\title{
Angiotensin receptor blockers for heart failure
}

\author{
Phillip Jong ${ }^{1}$, Catherine Demers ${ }^{2}$, Robert S McKelvie ${ }^{3}$, and Peter Liu ${ }^{4}$ \\ ${ }^{1}$ University of Toronto, Toronto, Canada. \\ ${ }^{2}$ HHSC - General Hospital, McMaster Clinic 3rd Floor, Hamilton, Canada. \\ ${ }^{3}$ Heart Function Clinic, Hamilton Health Sciences Corporation, Hamilton, Canada. \\ ${ }^{4}$ Toronto General Hospital, University of Toronto, Toronto, Canada
}

\begin{abstract}
This is the protocol for a review and there is no abstract. The objectives are as follows:

- To determine the efficacy of ARBs on the survival of patients with heart failure when compared to placebo or ACEIs.

- To determine the efficacy of ARBs on hospitalization rates in patients with heart failure when compared to placebo or ACEIs.
\end{abstract}

\section{BACKGROUND}

\begin{abstract}
Randomized controlled trials totaling more than 10,000 patients have shown angiotensin converting enzyme inhibitors (ACEIs) to benefit patients with heart failure in reducing mortality and hospitalization (Flather 2000). The favorable effects of ACEIs are partly attributed to the blockade of the production of angiotensin II from angiotensin I through the inhibition of the angiotensin converting enzymes by ACEIs (Lonn 1994), which is further augmented by the retarded degradation of vasodilating hormone such as bradykinin (Davie 1999). Angiotensin II is a potent vasoconstrictor, promotes vascular hypertrophy, and mediates aldosterone release (Ardaillou 1999). These biological effects impair ventricular function in patients with heart failure by causing volume overload, inducing ventricular hypertrophy, and promoting unfavorable ventricular remodeling (Weber 1996). In some patients with heart failure, however, this blockade of the renin-angiotensin-aldosterone system (RAAS) is incomplete. This is because even when the ACEI activity remains considerably suppressed after adequate ACEI dosing, angiotensin II level may return to
\end{abstract}

Copyright $\odot 2009$ The Cochrane Collaboration. Published by John Wiley \& Sons, Ltd.

Contact address: Phillip Jong, University of Toronto, 44 St. Joseph Street, Suite 1908, Toronto, M4Y 2W4, Canada.

philip.jong@sympatico.ca.

Editorial group: Cochrane Heart Group.

Publication status and date: Edited (no change to conclusions), published in Issue 1, 2009.

CONTRIBUTIONS OF AUTHORS Jong coordinated the project, co-formulated the study hypotheses, co-developed the search strategy, and co-drafted the initial protocol.

Demers co-formulated the study hypotheses, co-developed the search strategy, and revised the manuscript. McKelvie provided methodological support, acted as content expert, and revised the manuscript.

Liu provided methodological support, acted as content expert, and co-drafted the initial protocol.

DECLARATIONS OF INTEREST None known 
pretreatment level over time (Juillerat 1990). Patients in whom ACEIs fail to suppress angiotensin II have a poorer prognosis when compared to those in whom angiotensin II remains suppressed (Roig 2000; Swedberg 1990). This so-called "angiotensin escape" phenomenon may be related to the formation of angiotensin II through non-ACEI-dependent pathways by proteases such as chymases, cathepsin G, and chymostatin sensitive angiotensin II generating enzymes that are not inhibited by ACEIs (Urata 1990).

In contrast, angiotensin receptor blockers (ARBs) offer improved blockade of the RAAS over ACEIs by directly inhibiting the binding of angiotensin II to the AT1 (angiotensin II type 1) receptors, through which most deleterious cardiovascular effects of angiotensin II described above are believed to be mediated (Brunner-La 1999). This blockade by ARBs is irrespective of whether angiotensin II is produced systematically through the action of ACEdependent or alternate non-ACE-dependent pathways. In addition, blockade of the AT1 receptors by ARBs induces compensatory increases in plasma renin and angiotensin II. The resultant higher levels of angiotensin II in turn stimulate the AT2 (angiotensin II type 2) receptors that are not blocked by ARBs. This unopposed stimulation of the AT2 receptors may impart beneficial cardiac and vascular effects such as vasodilation, growth inhibition and regulation of apoptosis (Liu 1997). Furthermore, by not interfering with the degradation of bradykinin that is responsible for the side effects of ACEIs, ARBs appear to be better tolerated (Pitt 1997).

Despite the theoretical superiority of ARBs over ACEIs, the efficacy of ARBs in the treatment of heart failure remains unclear to date. Existing clinical data raise two possible therapeutic uses of ARBs in the heart failure population: 1) ARBs as substitute therapy to ACEIs, and 2) ARBs as adjunctive therapy to existing ACEIs. A systematic review of existing trial data may therefore provide new insights not seen in individual trials on the use of this drug class in patients with heart failure.

\section{OBJECTIVES}

- To determine the efficacy of ARBs on the survival of patients with heart failure when compared to placebo or ACEIs.

- To determine the efficacy of ARBs on hospitalization rates in patients with heart failure when compared to placebo or ACEIs.

\section{METHODS}

\section{Criteria for considering studies for this review}

Types of studies-Only published Phase II and III randomized controlled clinical trials enrolling patients with symptomatic heart failure with ARB as the experimental intervention will be considered.

Studies will be included if:

- Treatment assignments are randomized and administrated in parallel (i.e. no crossover). Studies with more than one ARB arm are allowed. Only blinded studies are permitted. Quasi-randomization scheme is accepted. 
- Mortality and/or hospitalization rates are/is reported as either clinical or safety endpoint(s). Studies may be counted even if no event of interest has occurred in both/either treatment and/or control group(s) if this is explicitly reported. Endpoints will be counted if they occur outside of the period of randomized therapies but within the follow-up period.

- Controlled interventions are either placebos or ACEIs. More than one control arm is allowed.

- Duration of randomized therapy is at least four weeks (i.e. studies in which treatment consists of only a single one-time dose of the ARB, such as in hemodynamic, pharmacodynamic dose-response, or safety studies, are excluded).

Studies will be excluded if:

- Protocol includes co-administration of other non-randomized investigational agents (e.g. angiotensin II, bradykinin). Studies with co-administration of other randomized investigational agents, such as beta-blockers in a nested partial factorial design, are allowed.

- Published only in abstract forms or non-peer reviewed journals whereby no further or insufficient information can be procured from the authors.

Types of participants-All included participants have symptomatic heart failure with New York Heart Association functional class II-IV. Asymptomatic subjects with left ventricular dysfunction are excluded. Echocardiographic, radionuclide, or angiographic documentation of ventricular dysfunction is not required if the clinical diagnosis of heart failure has been established by the study investigators.

Types of interventions-The experimental intervention is ARB. Studies with more than one control arm are allowed if at least one control intervention is either a placebo or an ACEI. If placebos are used as controls, both studies with and without background open-label ACEI therapy will be included. Only oral therapies are considered. The interventions can be administered as single agents, combination therapies (including ARB-ACEI combo), and in fixed or stepped/titrated doses.

Types of outcome measures-The primary outcome is all-cause mortality. The secondary outcome is hospitalization for heart failure. Tertiary outcomes include deaths from heart failure and all-cause hospitalization. We define hospitalization for heart failure as a hospital admission for worsening signs or symptoms of heart failure, for complications relating to the treatment of heart failure, or for syncope or arrhythmias related to heart failure. We define deaths from heart failure as deaths that are preceded by worsening signs or symptoms of heart failure, including cardiogenic shock. At present, reports of other outcomes of interest, such as cardiovascular deaths and hospitalization for cardiovascular causes, are erratic, and their inclusion may introduce selection bias.

\section{Search methods for identification of studies}

See: Collaborative Review Group search strategy 
We will conduct sensitive electronic searches of MEDLINE (1966 to January 2001), EMBASE (1980 to January 2001), Biological Abstracts (1980 to January 2001), International Pharmaceutical Abstracts (1970 to January 2001), and the Cochrane Controlled Trials Database (up to 2000 Issue 4). The McMaster CVD Trials Registry and personal files will be searched. Science Citation Index (1993 to January 2001) will be searched for additional trials that have made references to relevant studies found by the above searches. No language restriction will be applied.

The computer search of MEDLINE will use a search strategy based on the Collaborative Review Group search strategy for CD-PLUS OVID (Version 3.0) (Clarke 1999) plus the following:

1. exp Heart failure, congestive/

2. cardiomyopath:.tw.

3. (chf or hf).tw.

4. (heart adj25 failure).tw.

5. (cardiac adj25 (failure or insufficiency)).tw.

6. or/1-5

7. exp Receptors, angiotensin/

8. exp Losartan/

9. $\operatorname{arb}$ ?.tw.

10. (angiotensin: adj25 receptor: adj25 (block: or antagon: or inhibit:)).tw.

11. (candesartan or elisartan or embusartan or eprosartan or forasartan or irbesartan or losartan or saprisartan or tasosartan or telmisartan or valsartan or zolasartan).tw.

12. (139481-59-7 or $145040-37-5$ or $133040-01-4$ or $145216-43-9$ or $138402-11-6$ or $114798-26-4$ or $124750-99-8$ or $146613-90-3$ or $146623-69-0$ or $145733-36-4$ or 144701-48-4 or 137862-53-4 or 145781-32-4).rn.

13. or $/ 7-12$

14. 6 and 13

The computer search of EMBASE will use an EMBASE Randomized Controlled Trial filter (Lefebvre 1996) plus the following:

\#1 explode 'heart failure' / all subheadings

\#2 explode 'congestive cardiomyopathy' / all subheadings

\#3 cardiomyopath*

\#4 chf or hf

\#5 heart and failure

\#6 cardiac and (failure or insufficiency) 
$\# 7 \# 1$ or \#2 or \#3 or \#4 or \#5 or \#6

\#8 explode 'angiotensin receptor antagonist' / all subheadings

\#9 arb?

\#10 angiotensin* and receptor* and (block* or antagon* or inhibit*)

\#11 candesartan or elisartan or embusartan or eprosartan or forasartan or irbesartan or losartan or saprisartan or tasosartan or telmisartan or valsartan or zolasartan

\#12 (139481-59-7 or $145040-37-5$ or $133040-01-4$ or $145216-43-9$ or $138402-11-6$ or $114798-26-4$ or $124750-99-8$ or $146613-90-3$ or $146623-69-0$ or $145733-36-4$ or $144701-48-4$ or $137862-53-4$ or $145781-32-4)$ in rn

$\# 13$ \#8 or \#9 or \#10 or \#11 or \#12

\#14 \#7 and \#13

The computer search of Biological Abstracts and International Pharmaceutical Abstracts will use the following search strategy:

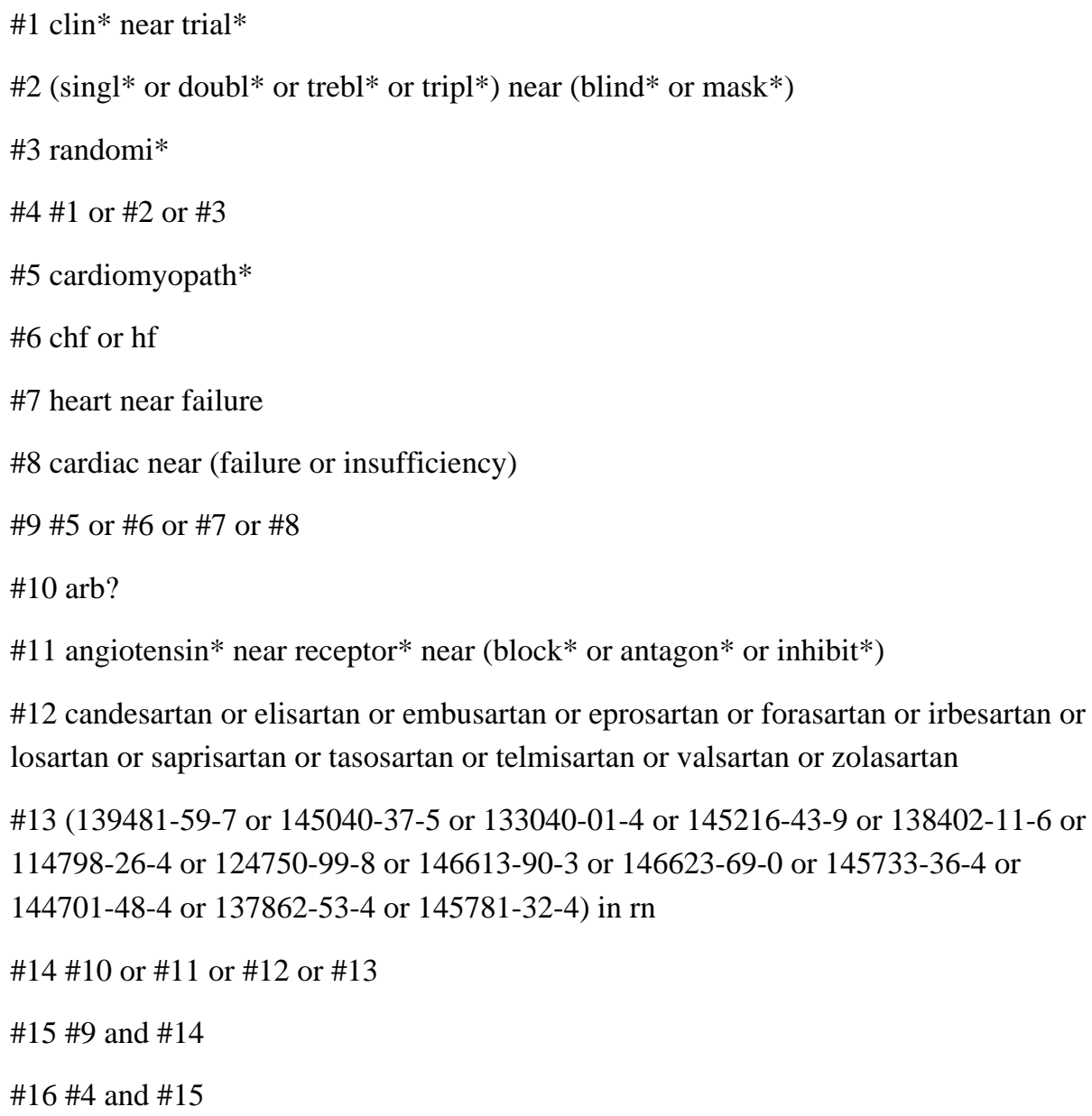

The computer search of the Cochrane Controlled Trials Database will use the following search strategy: 


\section{HEART-FAILURE-CONGESTIVE*:ME}

\section{CARDIOMYOPATH*}

(CHF or HF)

(HEART and FAILURE)

(CARDIAC and FAILURE)

(CARDIAC and INSUFFICIENCY)

(((((\#1 or \#2) or \#3) or \#4) or \#5) or \#6)

RECEPTORS-ANGIOTENSIN*:ME

LOSARTAN $*$ ME

(ARB or ARBS)

(ANGIOTENSIN* and (RECEPTOR* and ((BLOCK* or ANTAGON*) or INHIBIT*)))

$(((()((()((C A N D E S A R T A N$ or ELISARTAN) or EMBUSARTAN) or EPROSARTAN) or FORASARTAN) or IRBESARTAN) or LOSARTAN) or SAPRISARTAN) or TASOSARTAN) or TELMISARTAN) or VALSARTAN) or ZOLASARTAN)

((((\#8 or \#9) or \#10) or \#11) or \#12)

(\#7 and \#13)

Bibliographies of relevant studies will be screened for additional trials. Content experts in heart failure will be consulted to identify hard-to-find studies missed by our search strategies as well as follow-ups on trials for which only abstracts have so far been published. Pharmaceutical companies will be contacted to clarify published data.

We are aware of only one meta-analysis (Sharma 2000) that has been published on ARBs for heart failure. Studies identified from that paper will be included.

\section{Data collection and analysis}

Relevant trials will be determined by consensus between four reviewers from all studies identified from the above searches, using a pre-tested form that outlines key reasons for exclusion of the irrelevant studies.

Outcome data will be abstracted using a pre-tested data extraction form. Outcome measures reported are expected to differ among the trials. Furthermore, not all trials will report both mortality and hospitalization events. Definitions of cause-specific mortality and hospitalization are expected to differ between studies, and not all studies may provide adequate descriptions for these events to verify compatibility with our own definitions. In cases where outcome definitions are ambiguous, only events which we think most closely match our definitions will be pooled.

Scoring scales will not be used to assess the methodological quality of the trials because of their poor reliability in identifying trials of high from low quality (Juni 1999). Instead, the 
adequacy of random allocation, blinding, and descriptions of withdrawals and dropouts will be individually judged for each trial.

Data abstraction and quality assessment will be independently completed by two reviewers. Disagreements will be resolved by consensus and/or a third reviewer.

Analysis-All analyses of outcomes will be based on intention-to-treat principle. Crude treatment effects on mortality and hospitalization for each study will be reported as standard odds ratios. Pooled odds ratios across all studies will be calculated based on the MantelHaenszel method (Mantel 1959) for fixed effects models and the methods of DerSimonian \& Laird (DerSimonian 1986) for random effects models, weighted according to the inverse of the variance of each study. Chi-square test for heterogeneity will be used to assess the pooled outcome data for compatibility with the assumption of a fixed effects model ( $>>0.10)$. The $95 \%$ confidence intervals will be reported for all effect sizes for both individual trial and pooled estimates, the latter based on a general estimator for the variance of the odds ratio under sparse data (Robins 1986). Studies in which no event of interest is registered in both/either treatment and/or control groups will still be included in the analysis, but a continuity correction factor (Fleiss 1981) of $1 / 2$ will be added in these cases to the event numbers in order to avoid division by zero when calculating the odds ratio.

In our primary analysis, all ARBs will be combined regardless of dosages assuming a class effect and analyzed regardless of the types of controls (placebo or ACEI). The latter strategy has been used in a previous meta-analysis (Sharma 2000) of losartan in heart failure. The analysis is considered conservative, given that the inclusion of ACEIs, with a confirmed mortality benefit in heart failure, will only tend to underestimate the true treatment benefit of ARBs when compared against placebo controls. Combination therapy with ARBs and ACEIs will be analyzed "as" the ARB arm and compared against controls. For studies with more than one control arm (such as both placebo and ACEI), all controls will be combined to form one "mixed" control arm and compared against the ARB arm. Given the heterogeneity in the types of controls used, random effects models will be used to report the primary analysis.

Secondary stratified analyses will be conducted to refine the types of treatment comparison. Three treatment comparisons will be made: 1) ARBs versus placebos, without background ACEI therapy 2) ARBs versus ACEIs, and 3) combination therapy of ARBs and ACEIs versus ACEI alone. The latter comparison of combination therapy with ARBs and ACEIs against ACEIs alone is methodologically similar to a comparison of ARBs against placebos where background (open-label) ACEI therapy is given. Trials with these latter two designs will be categorized as making the same type of treatment comparison. Fixed effects models will be used to report all stratified analyses, unless the chi-square tests for heterogeneity suggest otherwise.

Sensitivity analysis for all outcome measures will be conducted by assuming both fixed effects model (Fleiss 1981) and random effects model for the pooled treatment effects of ARBs. Stratified analyses will be compared to the primary analysis to determine whether any observed heterogeneity in the treatment effects of ARBs is partly due to differences in 
the controls (placebos or ACEIs) against which ARBs are compared. Pooled effect estimates will be recalculated using the Peto odds ratio method (Yusuf 1985) and then compared against those calculated by the methods of DerSimonian \& Laird. We recognize that the Peto method may yield biased results when applied to unbalanced data that may be seen in our pooled comparisons (Greenland 1990). Pooled effect estimates will also be recalculated after exclusion of 1) poor quality studies or 2) studies with less than 6 months of randomized treatment. The latter criteria will exclude those trials in which the treatment duration may be too short for the intervention to modify our outcomes of interest even when the intervention is truly efficacious. In all scenarios, each pooled treatment effect of ARBs will be considered "qualitatively" robust if the upper and lower confidence bounds for the pooled treatment effect remain unchanged in direction with respect to unity. A funnel plot (Egger 1997) of all included trials will be used to check for the presence of publication bias.

\section{Acknowledgments}

Laura A. Demopoulos (Merck Frosst), Boriana Parvez (Toronto), Federico Zaglia (Toronto)

\section{SOURCES OF SUPPORT}

Internal sources

- University of Toronto, Canada.

- McMaster University, Canada.

- Hamilton Health Sciences Corporation, Canada.

- Heart and Stroke Research Organisation of Canada, Canada.

External sources

- $\quad$ No sources of support supplied

\section{HISTORY}

Protocol first published: Issue 2, 2001

\section{WHAT'S NEW}

\begin{tabular}{lll}
\hline Date & Event & Description \\
\hline 8 September 2008 & Amended & Converted to new review format. \\
\hline
\end{tabular}

\section{Additional references}

Ardaillou 1999 . Ardaillou R. Angiotensin II receptors. J Am Soc Nephrol. 1999; 10(Suppl 11):S30S39. [MEDLINE: 87]. [PubMed: 9892138]

Brunner-La 1999 . Brunner-La Rocca HP, Vaddadi G, Esler MD. Recent insight into therapy of congestive heart failure: focus on ACE inhibition and angiotensin-II antagonism. J Am Coll Cardiol. 1999; 33(5):1163-1173. [MEDLINE: 81]. [PubMed: 10193712]

Clarke 1999 . Clarke, M.; Oxman, AD. Review Manager (RevMan). Version 4.0. The Cochrane Collaboration; Oxford, England: 1999. Cochrane Reviewers' Handbook 4.0. [MEDLINE: 90200]

Davie 1999 . Davie AP, Dargie HJ, McMurray JJ. Role of bradykinin in the vasodilator effects of losartan and enalapril in patients with heart failure. Circulation. Jul 20; 1999 100(3):268-273. [MEDLINE: 95]. [PubMed: 10411851] 
DerSimonian 1986 . DerSimonian R, Laird N. Meta-analysis in clinical trials. Control Clin Trials. 1986; 7(3):177-188. [MEDLINE: 90116]. [PubMed: 3802833]

Egger 1997 . Egger M, Davey SG, Schneider M, Minder C. Bias in meta-analysis detected by a simple, graphical test. BMJ. Sep 13; 1997 315(7109):629-634. [MEDLINE: 90212]. [PubMed: 9310563]

Flather 2000 . Flather MD, Yusuf S, Kober L, Pfeffer M, Hall A, Murray G, Torp-Pedersen C, Ball S, Pogue J, Moye L, Braunwald E. Long-term ACE-inhibitor therapy in patients with heart failure or left- ventricular dysfunction: a systematic overview of data from individual patients. ACE-Inhibitor Myocardial Infarction Collaborative Group. Lancet. Jun 5; 2000 355(9215):15751581. [MEDLINE: 312]. [PubMed: 10821360]

Fleiss 1981 . Fleiss, JL. Statistical methods for rates and proportions. John Wiley \& Sons; New York: 1981. p. 2[MEDLINE: 110051]

Greenland 1990 . Greenland S, Salvan A. Bias in the one-step method for pooling study results. Stat Med. 1990; 9(3):247-252. [MEDLINE: 90115]. [PubMed: 2343220]

Juillerat 1990 . Juillerat L, Nussberger J, Menard J, Mooser V, Christen Y, Waeber B, Graf P, Brunner HR. Determinants of angiotensin II generation during converting enzyme inhibition. Hypertension. 1990; 16(5):564-572. [MEDLINE: 10051]. [PubMed: 2172161]

Juni 1999 . Juni P, Witschi A, Bloch R, Egger M. The hazards of scoring the quality of clinical trials for meta-analysis. JAMA. Sep 15; 1999 282(11):1054-1060. [MEDLINE: 90246]. [PubMed: 10493204]

Lefebvre 1996 . Lefebvre, C.; McDonald, S. Development of a sensitive search strategy for reports of randomized controlled trials in EMBASE. The Fourth International Cochrane Colloquium; Adelaide, Australia. 20-24 Oct.; 1996. [MEDLINE: 90214]

Liu 1997 . Liu YH, Yang XP, Sharov VG, Nass O, Sabbah HN, Peterson E, Carretero OA. Effects of angiotensin-converting enzyme inhibitors and angiotensin II type 1 receptor antagonists in rats with heart failure. Role of kinins and angiotensin II type 2 receptors. J Clin Invest. Apr 15; 1997 99(8):1926-1935. [MEDLINE: 82]. [PubMed: 9109437]

Lonn 1994 . Lonn EM, Yusuf S, Jha P, Montague TJ, Teo KK, Benedict CR, Pitt B. Emerging role of angiotensin-converting enzyme inhibitors in cardiac and vascular protection. Circulation. 1994; 90(4):2056-2069. [MEDLINE: 8]. [PubMed: 7923694]

Mantel 1959 . Mantel N, Haenszel W. Statistical aspects of the analysis of data from retrospective studies of disease. J Natl Cancer Inst. 1959; 22:719-748. [MEDLINE: 110035]. [PubMed: 13655060]

Pitt 1997 . Pitt B, Segal R, Martinez FA, Meurers G, Cowley AJ, Thomas I, Deedwania PC, Ney DE, Snavely DB, Chang PI. Randomised trial of losartan versus captopril in patients over 65 with heart failure (Evaluation of Losartan in the Elderly Study, ELITE). Lancet. Mar 15; 1997 349(9054):747-752. [MEDLINE: 86]. [PubMed: 9074572]

Robins 1986 . Robins J, Greenland S, Breslow NE. A general estimator for the variance of the Mantel-Haenszel odds ratio. Am J Epidemiol. 1986; 124(5):719-723. [MEDLINE: 110053]. [PubMed: 3766505]

Roig 2000 . Roig E, Perez-Villa F, Morales M, Jimenez W, Orus J, Heras M, Sanz G. Clinical implications of increased plasma angiotensin II despite ACE inhibitor therapy in patients with congestive heart failure. Eur Heart J. 2000; 21(1):53-57. [MEDLINE: 78]. [PubMed: 10610744]

Sharma 2000 . Sharma D, Buyse M, Pitt B, Rucinska EJ. Meta-analysis of observed mortality data from all-controlled, double-blind, multiple-dose studies of losartan in heart failure. Am J Cardiol. 2000; 85(2):187-192. [MEDLINE: 84]. [PubMed: 10955375]

Swedberg 1990 . Swedberg K, Eneroth P, Kjekshus J, Wilhelmsen L. Hormones regulating cardiovascular function in patients with severe congestive heart failure and their relation to mortality. CONSENSUS Trial Study Group. Circulation. 1990; 82(5):1730-1736. [MEDLINE: 10]. [PubMed: 2225374]

Urata 1990 . Urata H, Kinoshita A, Misono KS, Bumpus FM, Husain A. Identification of a highly specific chymase as the major angiotensin II- forming enzyme in the human heart [published erratum appears in J Biol Chem 1991 Jun 25;266(18):12114]. J Biol Chem. Dec 25; 1990 265(36):22348-22357. [MEDLINE: 79]. [PubMed: 2266130] 
Weber 1996 . Weber KT, Sun Y, Katwa LC. Wound healing following myocardial infarction. Clin Cardiol. 1996; 19(6):447-455. [MEDLINE: 88]. [PubMed: 8790947]

Yusuf 1985 . Yusuf S, Peto R, Lewis J, Collins R, Sleight P. Beta blockade during and after myocardial infarction: an overview of the randomized trials. Prog Cardiovasc Dis. 1985; 27(5): 335-371. [MEDLINE: 20031]. [PubMed: 2858114]

* Indicates the major publication for the study 